\title{
Resource Management in OFDMA Heterogeneous Network
}

\author{
Maria A. Seimeni ${ }^{1}$, Panagiotis K. Gkonis ${ }^{1}$, Dimitra I. Kaklamani ${ }^{1}$, Iakovos S. Venieris ${ }^{1}$ and Christos A. Papavasiliou ${ }^{2}$ \\ ${ }^{1}$ National Technical University of Athens, School of Electrical and Computer Engineering, \\ 9 Heroon Polytechneioy str, Zografou, Athens, Greece \\ ${ }^{2}$ Electrical and Electronic Engineering, Imperial College, South Kensington Campus, London SW7 2AZ, England \\ e-mails: mseimeni@icbnet.ntua.gr, pgkonis@esd.ntua.gr,dkaklam@mail.ntua.gr, venieris@cs.ntua.gr, c.papavas@imperial.ac.uk
}

\begin{abstract}
In this study, a Long Term Evolution Advanced (LTEa) - based multi-user Orthogonal Frequency Division Multiple Access (OFDMA) heterogeneous network has been simulated and a resource allocation strategy has been proposed. The strategy under consideration can inherently mitigate electromagnetic interference, hence increases the mean number of terminals, and requires no channel state information (CSI). To evaluate the performance of the network platform and the proposed strategy, the system is studied for different network orientations. According to the results, the platform is a good reality simulator, whereas owning to the proposed Radio Resource Management (RRM) algorithm the mean capacity can reach a 12-fold increase especially for highly noisy operating environments.
\end{abstract}

Keywords - Ite advanced; radio resource management; ofdm; simulation platform.

\section{INTRODUCTION}

The fast-moving emerging wireless technologies are mainly characterized by differences in hardware, software, network access scheme, resource demands and operating frequency bands (licensed, unlicensed). This multifaceted diversity along with the limited ubiquity between the wireless networks have led to a rather scarce spectrum and, now more than ever, service providers focus their interest on the heterogeneous networks (HetNets).

As the name implies, a HetNet consists of evolved Node Base stations (pico-/ femto-/ relay- eNBs), which transmit at diverse power levels and are typically deployed in an unplanned manner, and enables the mobile terminals (MTs) to experience the benefits of the complementary co-existence of a variety of traditional and new services. Currently, HetNets have been included in the LTEa [1], which is finalized by the 3GPP, and combined with Multiple Input Multiple Output arrays (MIMO [2]) and carrier aggregation (Orthogonal Frequency Division Multiplexing - OFDM [3]) to increase the spectral efficiency, the data rates (higher than 1 Gbps) and the throughput. In the same context, we develop an LTEa - based HetNet platform, which allows access to the MTs thanks to the OFDMA scheme, and focus our efforts on the enhancement of the mean overall capacity through judicious RRM.

As a baseband modulation scheme, OFDM is simple in principle and mainly for this reason it has been adopted by both wired [4] and wireless systems [5-6], and included in
IEEE 802.16x [7], IEEE 802.11x [8] and the LTEa standards [1]. With the OFDM, the available spectrum is divided into narrow flat fading frequency sub-bandwidths (subcarrier frequencies), hence it is robust to multipath fading, provides spectral efficiency and mitigates Intra-Cell Interference (ICI). If full frequency reuse is considered (FFR), the spectrum efficiency is further enhanced [9] at the cost, however, of the increased Co-Channel Interference (CCI) during downlink transmission (DL). Alternatively to the FFR, the fractional frequency reuse scheme can be considered. In this case, the cell is virtually partitioned in disjoint concentric sub-regions, each being assigned a different frequency reuse factor according to the channel condition. Towards this direction, RRM strategies are employed. Overall, the RRM strategies are distinguished to those which are based on game theory [10] or multiuser diversity [11], to those which exploit the CSI and those without channel feedback, and favor either throughput or power exploitation. In our study, multiuser diversity strategies without CSI are taken under consideration.

In [12], the base station allocates appropriately chunks of subcarriers to different users based upon a scaling factor and according to the estimated channel condition assigns bits per symbol and power per chunk. The results show that when dynamic power allocation is considered the mean capacity is better than the capacity achieved via the fixed power allocation scheme. In [13] the users are grouped according to their channel gains in those of a region close to the base station and to those close to the cell boundaries. The spectrum is likewise partitioned in two sets, each one assigned to the cell regions. This FFR-like strategy leads to increased data rates and mitigated ICI. Chang et al. [14] divide the cell area like [13] to improve system's capacity and data rates, while no CSI is considered at the receiver side. In [15], the cell is partitioned to mitigate ICI and improve the receivers' performance. To this end, the subcarriers are sequentially assigned and cell-edge receivers are provided with sufficient power. In [16], a delay-aware power and subcarrier allocation strategy are proposed to maximize effective energy efficiency, which is the ratio of the overall effective capacity to the total consumed power. In [17], the FFR scheme is evaluated in terms of the average number of bits that can be transmitted per symbol in the area and compared with the traditional frequency reuse. Results show that the FFR scheme can provide extra capacity, while it slightly penalizes the users at the cell edge. In [18], according to the CSI (the interference received by the neighboring eNBs is included) sent by each 
MT to the serving eNB, the eNB clusters the interfering eNBs and creates a wish list of resource blocks to be restricted in its neighboring cells. Simulations show that the proposed scheme outperforms the reference schemes in terms of throughput at the cell boundaries, while it lacks algorithmic simplicity.

However, some of the previously mentioned studies allocate resources in an exhaustive manner, while some others do not exploit the available spectrum efficiently. Trying to decrease algorithmic complexity, enhance spectral efficiency and increase networks capacity, we present the COOPERATIVE strategy and compare it to the traditional RANDOM [19], which to the best of our knowledge has been the most efficient strategy (very good spectrum exploitation, low complexity, increased throughput).

The outline of this paper is as follows: In section II, the network topology and the simulation platform are described. Section III presents the proposed RRM algorithm, while in the following section simulations are demonstrated for different traffic scenarios. In section V, this study is concluded.

\section{HeTNet PlatForm}

An LTEa-based HetNet for DL transmissions is studied. As Fig. 1 indicates, macro evolved Node Base stations (MacroeNBs) are located at the macrocell-centre, while each one is overlaid with one pico evolved Node Base station (Pico-eNB) so as to improve conditions in coverage. In this study, due to the computational complexity of the platform (section III), the positions of all Pico-eNBs are fixed for all simulation scenarios, whereas the dynamic positioning of the Pico-eNBs is included in our future studies. Both Macro- and Pico-eNBs have full RRM functionalities and are equipped with a threebeam directional antenna with radiation pattern (1) [2]:

$$
f(\varphi)=B G_{b}-\min \left[12\left(\frac{\varphi-\varphi_{k}}{\varphi_{3 d B}}\right)^{2}, A_{m}\right]
$$

for $\varphi_{k}-60^{\circ} \leq \phi \leq \phi_{k}+60^{\circ}$. In (1), $\varphi_{k} \in\left\{60^{\circ}, 180^{\circ}, 300^{\circ}\right\}$ is the pointing direction of the main lobe at sector $k, B G_{b}$ is the broadside antenna gain, $\varphi_{3 d B}$ is the $3-\mathrm{dB}$ beamwidth of the antenna pattern, and $A_{m}$ is the front-to-back ratio gain. The network topology is fully characterized by Table I.

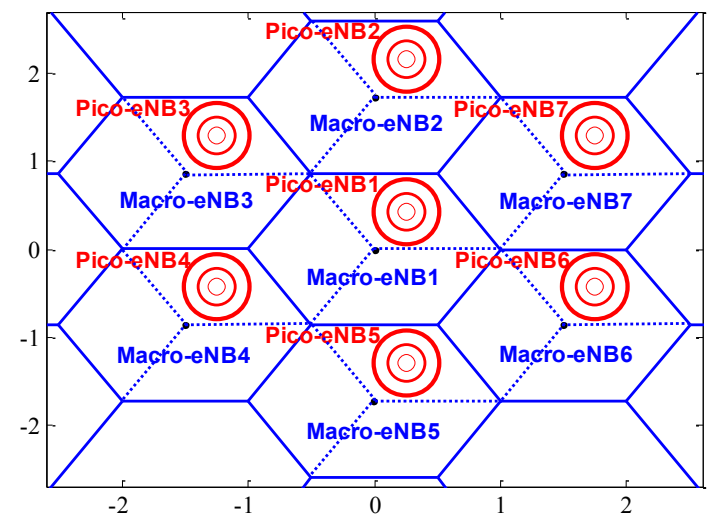

Fig. 1. Cellular configuration for 1 tier $(\mathrm{km})$.
TABLE I. SIMULATION PARAMETERS

\begin{tabular}{|c|c|}
\hline Number of Macro/ Pico cells & 7 \\
\hline Macro_/Pico_eNB height & $30 \mathrm{~m} / 30 \mathrm{~m}$ \\
\hline MT height & $1.5 \mathrm{~m}$ \\
\hline Propagation Model & COST 231 Hata model \\
\hline Standard deviation for shadowing & $8 \mathrm{~dB}$ \\
\hline Azimuth dispersion & Laplacian distribution, azimuth spread 5 deg \\
\hline Radiation pattern of the antenna element & $\begin{array}{c}\text { Macro-Broadside gain }=14 \mathrm{dBi} \\
\text { Pico-Broadside gain }=14 \mathrm{dBi}, \\
\text { 3-dB beamwidth }=70 \mathrm{deg} \\
\text { Front-to-back ratio }=20 \mathrm{~dB}\end{array}$ \\
\hline Subcarrier frequencies per MT & 2,3,4 (all possible combinations) \\
\hline Mobile Rx Sensitivity & $133.4 \mathrm{~dB}$ \\
\hline Failure Probability & $30 \%$ \\
\hline Number of MC simulations & 1000 \\
\hline Thermal noise level at Users (Inoise) & $-104 \mathrm{dBm}$ \\
\hline Macro SINR & $9.6 \mathrm{~dB}[17]$ \\
\hline Pico SINR & $5 \mathrm{~dB}$ or $9.6 \mathrm{~dB}$ \\
\hline Total subcarriers per sector (Macro /Pico) & $128 / 128$ \\
\hline
\end{tabular}

Once the HetNet surface is configured, the platform executes a predefined number (sufficient for the parameters of interest to converge to their mean value) of independent Monte Carlo (MC) simulations. During one MC, MTs do not change their location (semi-static platform), while a four-step procedure takes place:

- Step 1: Access port (cell) assignment;

- Step 2: Service request (either Macro- or Pico- eNB);

- Step 3: Waveform (subcarrier frequency) assignment; and

- Step 4: Transmission power assignment and power control.

In detail, during a MC run, MTs enter the system one at a time and are randomly located on the cellular lattice. Each $n^{\text {th }}$ MT $(1 \leq n \leq N)$ requests access to the nearest heterogeneous network $h n^{\text {th }}(1 \leq h n \leq H N)$ and pairs with the eNB (either Macro or Pico) with the lowest pathloss [20]. If the lowest path loss exceeds a predefined value $(133.4 \mathrm{~dB})$ the terminal is rejected, otherwise the total losses are estimated (shadowing and antenna radiation patterns are included) and the $b^{\text {th }}$ BS assigns without CSI a $U_{n}$ set of subcarrier frequencies. Subcarriers are assigned according to the algorithms that are presented in the following section. Then, a $p_{n, s}$ power level is assigned to each subcarrier frequency of the $n^{\text {th }}$ terminal (2):

$$
p_{n, s}=\left(\frac{E_{b}}{N_{o}}\right)_{n, s}\left(\sum_{k=1, k \neq i}^{K} P_{k, s} \frac{T L_{n, i}}{T L_{n, k}}+\mathrm{I}_{\text {noise }} T L_{n, i}\right)
$$

where $p_{n, s}$ is the transmission power for the $n^{\text {th }}$ MTs and $s^{\text {th }}$ subcarrier $(1 \leq s \leq S), K$ denotes the number of effective sectors, $i$ is the serving sector of the $n^{\text {th }}$ MT and $P_{k, s}$ is the total transmission power of the $k^{\text {th }}$ sector that has been allocated to the $s^{\text {th }}$ subcarrier. Moreover, $\mathrm{I}_{\text {noise }}$ is the total received thermal noise from the $n^{\text {th }}$ MT and $T L_{n, i}$ and $T L_{n, k}$ are the total losses of 
the $n^{\text {th }}$ MT for the $i$ and $k^{\text {th }}$ sectors respectively. $E_{b}$ is the bit energy and $N_{o}$ is the noise power spectral density. If $p_{n, s}$ power level exceeds 1 Watt (3a), the terminal is rejected and the process repeats for the next candidate terminal. It is, also, noted that each subcarrier frequency is assigned to no more than one terminal (3b).

$$
\begin{gathered}
0<p_{n, s} \leq 1, \quad 0<\sum_{s=1, s \in S_{n}} p_{n, s} \leq 1 \\
S_{n} \cap S_{i}=\varnothing, \quad \forall n, m \in N, \quad n \neq m
\end{gathered}
$$

$S_{n}$ indicates the number of subcarrier frequencies assigned to the $n^{\text {th }}$ MT. The MC simulation stops once the probability failure (number of active terminals per total number of access requests) exceeds a predefined value (herein $30 \%$ ).

\section{RAdio RESOURCE MANAGEMENT StRAtegIES}

This section describes the philosophy and defines the algorithmic complexity of the radio resource management algorithms. Since neither RANDOM, nor COOPERATIVE require channel feedback in order to allocate subcarrier frequencies, the complexity of the algorithms is quite straightforward and is also estimated. The COOPERATIVE is adopted by the Macro-eNBs only.

\section{A. RANDOM}

With this algorithm, the $b^{\text {th }} \mathrm{BS}$ assigns (at random) a set $U_{n}$ of available subcarriers $C_{b}$ to the $n^{\text {th }}$ accepted terminal at the $k^{\text {th }}$ sector.

$$
\begin{aligned}
& U_{n} \leftarrow \text { randsample }\left(S_{n}, C_{b}\right) \\
& C_{b} \leftarrow C_{b} \backslash U_{n}
\end{aligned}
$$

If $D$ is a set of elements, function randsample(i,D) returns at random a number $i$ of elements.

Supposing that the assignment of one subcarrier requires a full scan of all the subcarriers of the base station, then the complexity of RANDOM equals the product of the numbers of steps during one scan with the number $S_{n}$; hence $O\left(\left|C_{b}\right|{ }^{*} S_{n}\right)$.

\section{B. COOPERATIVE}

According to the algorithm (5), the $n^{\text {th }}$ MT must not reuse subcarriers which are "virtually" assigned to Interferers, that is users of neighboring BSs within a certain Range (herein 1.5 $\mathrm{km}$ ) from $n^{t h}$ MT, Fig. 2. If all the available subcarriers of the $b^{\text {th }} \mathrm{BS}$ are "engaged" by Interferers, $n^{\text {th }} \mathrm{MT}$ has to reserve subcarriers according to $R A N D$.

$$
\begin{aligned}
& \text { if } C_{b} \cap C J^{*}=\varnothing \\
& \quad C_{b} \leftarrow C_{b} \\
& \text { else } \\
& \qquad C_{b} \leftarrow C_{b} \cap C J^{*} \\
& \text { end } \\
& U_{n} \leftarrow \operatorname{rand}\left(S_{n}, C_{b}\right) \\
& C_{b} \leftarrow C_{b} \backslash U_{n}
\end{aligned}
$$

where $C J$ corresponds to the sets of subcarriers which are engaged by the Interferers and $C J^{*}$ are the sets of subcarriers which are not assigned to the Interferers.

In the following Venn Diagram, Fig. 2, an example is provided. In Fig. $2 \mathrm{a}$, the set $C_{1}$ (solid line circle) is the set of the available subcarriers of the $1^{\text {st }} \mathrm{BS}(b=1)$ that serves the $n^{\text {th }}$ accepted terminal, whereas the dashed line circles represent the set $C J_{j}$ (where $2 \leq j \leq 7$ ) of the subcarriers that are engaged by the Interferers. In detail, $C J_{3}$ is the set of the subcarriers that are actually assigned to the terminals served by the $3^{\text {rd }}$ BS and are $1.5 \mathrm{Km}$ far from the $n^{\text {th }} \mathrm{MT}$, etc.. It is also noted that $\mathrm{C}_{\mathrm{I}}$ is overlapped by $\mathrm{CJ}_{2}, \mathrm{CJ}_{4}$ and $\mathrm{CJ}_{7}$, which means that some of the available subcarriers of the $1^{\text {st }}$ BS are reused by some terminals of the neighboring BSs (in our case, one BS can be surrounded by six BSs at maximum). Therefore, when the COOPERATIVE strategy is employed, the $n^{\text {th }}$ MT has to be assigned a set of subcarriers of the visible part of $C_{l}$ indicated as $C^{\prime}{ }_{1}$ in Fig. $2 \mathrm{~b}$. The set $U_{n}$ of the subcarriers that are finally prepared for $D L$, Fig. $2 \mathrm{c}$, is a subset of $C_{l}$, while its intersection with the $C J_{q}$ sets is an empty set, hence:

$$
U_{n} \subseteq C_{1}^{\prime} \subseteq C_{1}, \quad U_{n} \cap C J_{j}=\varnothing
$$

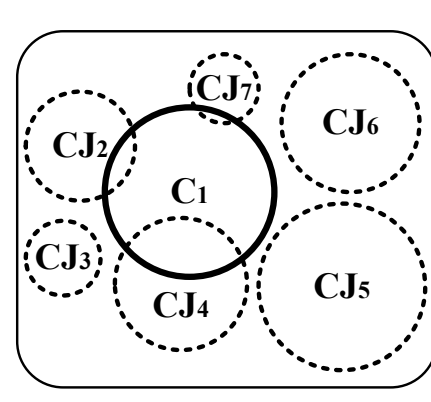

(a)

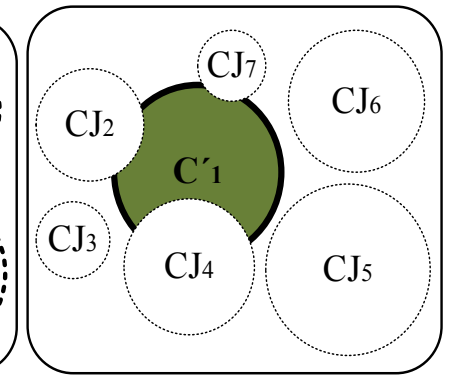

(b)

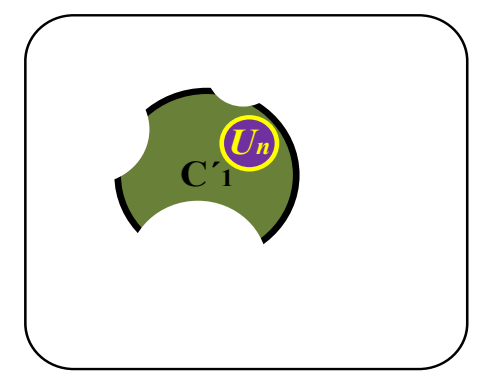

(c)

Fig. 2. Venn diagrams showing (a) the available set $C_{l}$ of subcarriers of the $1^{\text {st }} \mathrm{BS}$ and the subcarriers $C J_{q}$ engaged by the Interferers of the neighboring BSs, (b) the subcarriers $C^{\prime}{ }_{I}$ of the $1^{\text {st }} \mathrm{BS}$ that are available for DL when the COOPERATIVE strategy is adopted and (c) the set $U_{n}$ of the subcarriers of the $1^{\text {st }} \mathrm{BS}$ that are prepared for DL.

The algorithmic complexity of COOPERATIVE equals $O\left((J+1)\left|C_{b}\right| * S_{n}\right)$, where $J$ is the number of neighboring BSs which serve the Interferers. Therefore, (in the worst case scenario, hence $J=6$ ) the subcarrier frequencies of the $J$ neighboring base stations and the serving BS must be scanned, which leads to a preliminary complexity $O\left((J+1) * S_{n}\right)$. The procedure repeats for all the $U_{n}$ number of subcarrier frequencies resulting in a $O\left((J+1) S_{n}{ }^{*} U_{n}\right)$ complexity. 


\section{SimULATIONS}

In simulations, we consider two scenarios. In the first scenario, both Macro- and Pico-eNBs adopt RANDOM algorithm (RAND-RAND, sub-figures (a)), while in the second COOPERATIVE and RANDOM (COOP-RAND, subfigures (b)) respectively. In all graphs, there are two groups of bars, each one representing either Macro- or Pico- eNB and their respective resource allocation strategy. Furthermore, each bar corresponds to the subcarriers which are assigned to the terminals. If for example the Macro-eNB assigns 3 subcarriers to each MT and the Pico-eNB assigns 2 subcarriers, then the resource allocation set is indicated with the seventh bar $((3,2)$ in the text-box of the plots). Figures 3 to 6 reflect the HetNet performance for 7 eNBs (either Macro or Pico), while the rest figures represent a 19 eNBs HetNet topology.

Overall, as the number of subcarriers decreases, the mean capacity of the HetNet increases, as it can be observed from Figures 3, 5, 7 and 8. Alternatively, the capacity increases when the SINR is low (herein $5 \mathrm{~dB}$ ). More precisely, in Fig. 3a (or Fig. 3b), both eNBs behave identically for the same number of subcarriers per MT, while they support a different number of MTs for the rest cases. Likewise in Fig. 3, the same tendency is shown in Fig. 5. It is also obvious that COOP$R A N D$ performs roughly the same as $R A N D-R A N D$.

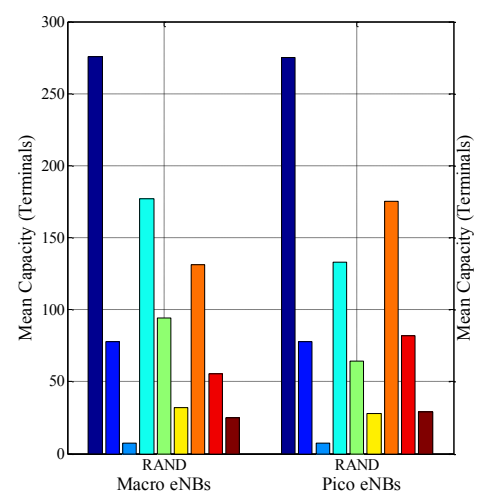

(a)

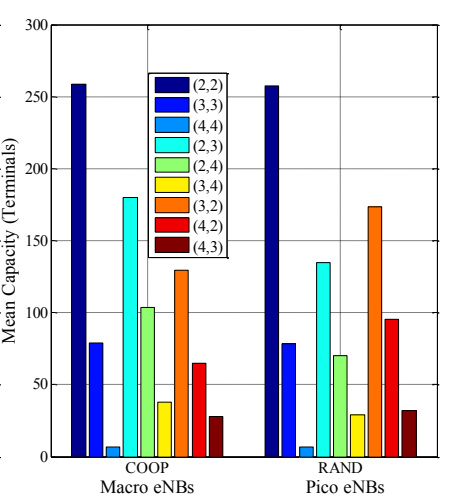

(b)
Fig. 3. Mean HetNet capacity (MTs) for (a) RAND-RAND and (b) COOPRAND scenarios. [SINR Macro-eNB $^{-}$SINR $_{\text {Pico-eNB }}=9.6 \mathrm{~dB}-9.6 \mathrm{~dB}$ ], 7 eNBs.

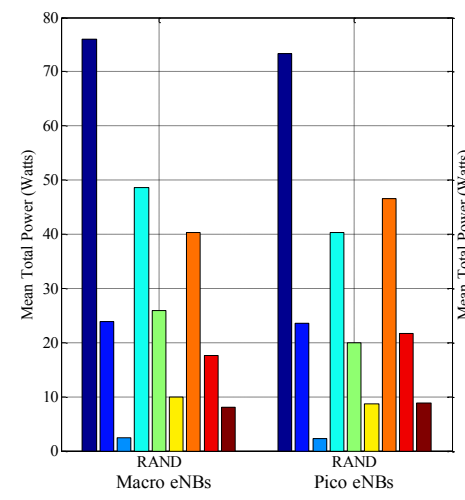

(a)

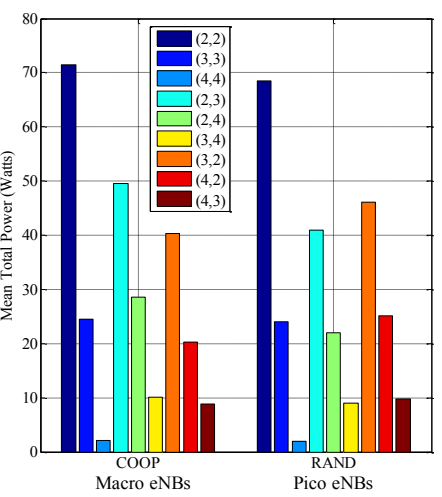

(b)
Fig. 4. Mean HetNet dissipated power (MTs) for (a) RAND-RAND and (b) COOP-RAND scenarios. $\left[\mathrm{SINR}_{\mathrm{Macro-eNB}}-\mathrm{SINR}_{\text {Pico-eNB }}=9.6 \mathrm{~dB}-9.6 \mathrm{~dB}\right], 7$ eNBs.

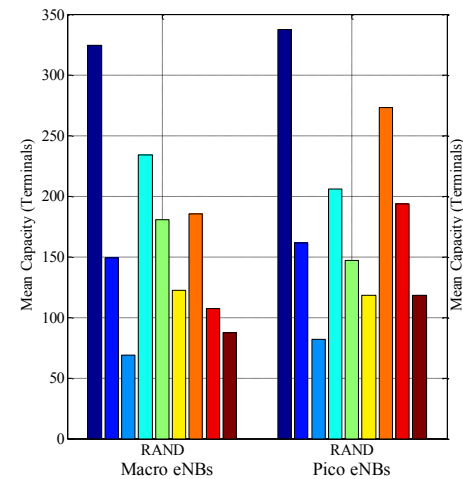

(a)

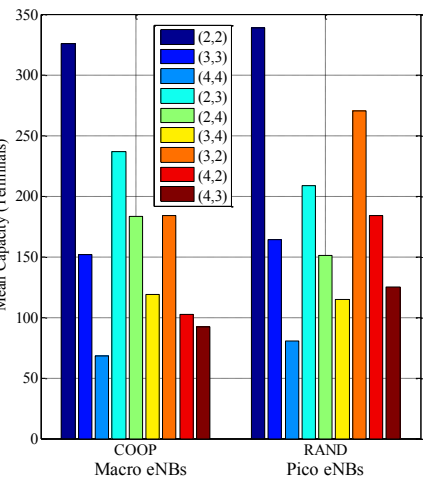

(b)
Fig. 5. Mean HetNet capacity (MTs) for (a) RAND-RAND and (b) COOPRAND scenarios. $\left[\right.$ SINR $_{\text {Macro-eNB }}-$ SINRR $\left._{\text {Pico-eNB }}=9.6 \mathrm{~dB}-5 \mathrm{~dB}\right], 7$ eNBs.

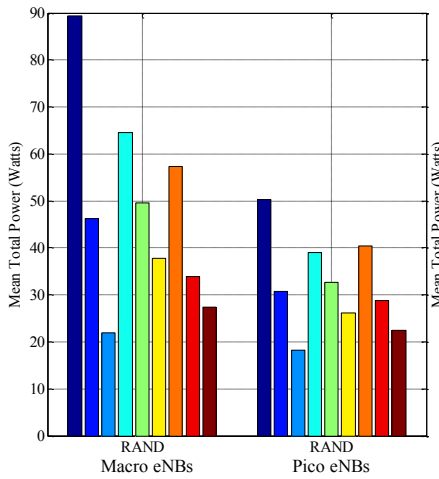

(a)

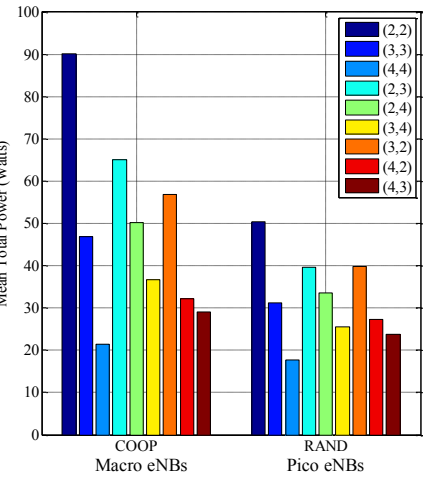

(b)
Fig. 6. Mean HetNet dissipated power (MTs) for (a) RAND-RAND and (b) COOP-RAND scenarios. $\left[\mathrm{SINR}_{\mathrm{Macro}-\mathrm{eNB}}-\mathrm{SINR}_{\text {Pico-eNB }}=9.6 \mathrm{~dB}-5 \mathrm{~dB}\right], 7$ eNBs.

For 19 eNBs (Fig. 7, 8, 9), COOPERATIVE differentiates its efficiency from RAND. In this case, as the overall capacity tends to increase, the inherent anti-jamming property of the strategy now makes sense (the number of Interferers is higher than their number when only one tier is considered). This means that the COOPERATIVE can mitigate the electromagnetic interference (EMI) especially in these highly noisy environments. In detail for the Macro-eNBs (Fig. 7), where both eNBs present equal SINR values, it is obvious that the COOPERATIVE not only increases the mean capacity in the macro-cell, but also indirectly boosts the mean capacity in the pico-cell as well. The capacity gain (\%) for this case is provided in Fig. 9a. With respect to Fig. 7a (or Fig. 7b), in Fig. $8 \mathrm{a}$ (or Fig. $8 \mathrm{~b}$ respectively), the $\mathrm{SINR}_{\text {pico-eNB }}$ deteriorates down to $5 \mathrm{~dB}$ and as a result the capacity decreases for both eNBs. However, when the COOPERATIVE is implemented, Fig. 8b, the gain capacity with reference to Fig. 8a can reach over a $12-$ fold increase (Fig. 9) as in the case of three subcarriers per MT for both eNBs (solid circles). Finally, it is noted that Fig. 9a corresponds to Fig. 7, whereas Fig. 9b corresponds to Fig. 8.

As for the mean dissipated power per network orientation, a high mean capacity leads to a high power consumption. When the SINR is low, Fig. 6, the power increases by roughly 15 Watts. This is expected, because in this case the MTs have to strengthen their signal in order to establish/maintain a 
connection to either sub-networks. Moreover, since a MacroeNB typically transmits at power level between $5 \mathrm{~W}$ and $40 \mathrm{~W}$ (35W up to $280 \mathrm{~W}$ for seven Macro-eNBs) approximately, and a Pico-eNB typically transmits at power level between $100 \mathrm{~mW}$ and $2 \mathrm{~W}$ ( $700 \mathrm{~mW}$ up to $14 \mathrm{~W}$ for seven Pico-eNBs) approximately, it is deduced that the power consumed by the platform is acceptable. For two tiers of eNBs, the dissipated power is scaled upwards and for this reason we have included results only for one tier, which are rather representative.

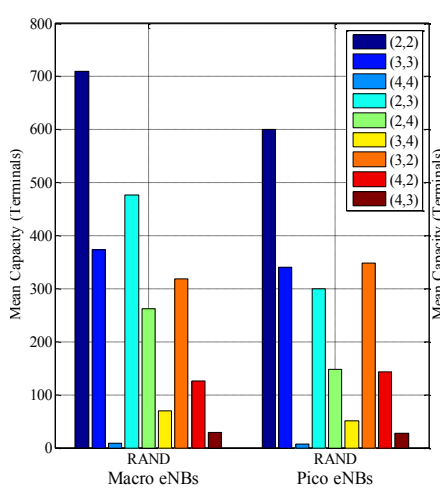

(a)

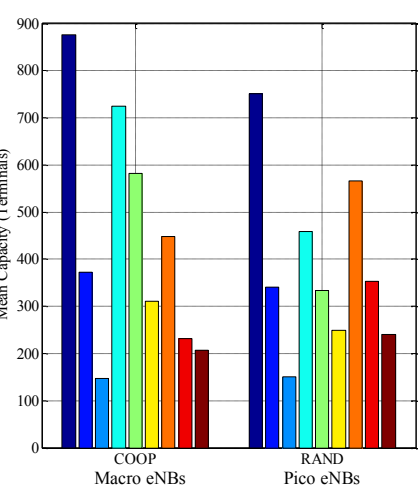

(b)
Fig. 7. Mean HetNet capacity (MTs) for (a) RAND-RAND and (b) COOPRAND scenarios. [SINR Macro-eNB - SINR $_{\text {Pico-eNB }}=9.6 \mathrm{~dB}-9.6 \mathrm{~dB}$ ], 19 eNBs.

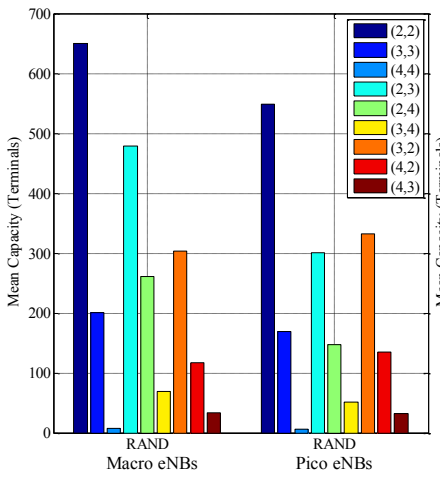

(a)

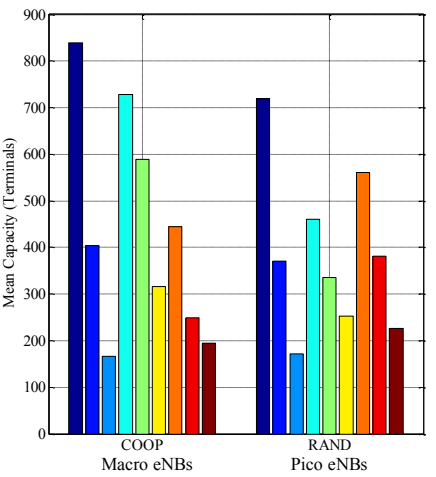

(b)
Fig. 8. Mean HetNet capacity (MTs) for (a) RAND-RAND and (b) COOPRAND scenarios. $\left[\right.$ SINR $_{\text {Macro-eNB }}-$ SINR $_{\text {Pico-eNB }}=9.6 \mathrm{~dB}-5 \mathrm{~dB}$ ], 19 eNBs.

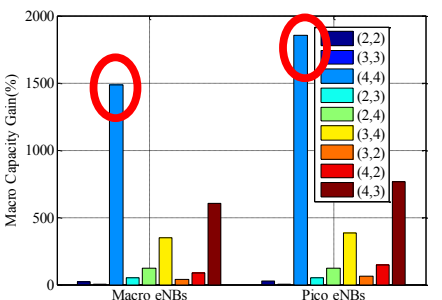

(a)

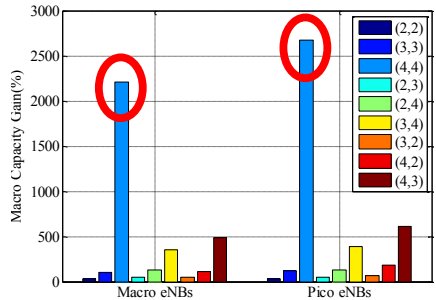

(b)
Fig. 9. Capacity gain (\%) in Macro- and Pico- eNBs owning to COOP, for (a) $\mathrm{SINR}_{\text {Macro-eNB}} / \mathrm{SINR}_{\text {Pico-eNB }}=9.6 \mathrm{~dB} / 9.6 \mathrm{~dB}$ and (b) $\mathrm{SINR}_{\text {Macro-eNB }} /$ SINR $_{\text {Pico-eNB }}=9.6 \mathrm{~dB} / 5 \mathrm{~dB}, 19$ eNBs.

\section{CONCLUSIONS}

In this paper, we studied an LTEa-based network, which consists of Macro- and Pico-eNBs. For this reason, a software platform is developed which simulates the network topology, assigns the available (spectrum and power) resources to the terminals and can support RRM strategies with or without CSI. From power simulations, it is first of all justified that the platform can be employed for practical examples as well. Simulation results on mean capacity show that the COOPERATIVE strategy outperforms RANDOM in highly noisy environments (19 eNBs). Inherently, COOPERATIVE combats EMI by allocating to a terminal those subcarriers which are not reused by Interferers (terminals of neighboring BSs within a certain Range). Consequently, COOPERATIVE leads to a capacity gain higher than this of RANDOM (an increase by 12 times).

Future work upgrades the platform with MIMO antennas and AS schemes, suggests new RRM strategies and evaluates Bit Error Rate (BER) for different hardware complexity. Ongoing work also includes dynamic positioning of the PicoeNBs.

\section{ACKNOWLEDGMENTS}

This research has been co-financed by the European Union (European Social Fund - ESF) and Greek national funds through the Operational Program "Education and Lifelong Learning" of the National Strategic Reference Framework (NSRF)-Research Funding Program: THALIS - NTUA, "Novel Transmit and Design Techniques for Broadband Wireless Networks" (MIS 379489).

\section{REFERENCES}

[1] 3GPP, TR 36.913, "Requirements for Further Advancements for EUTRA (LTE-Advanced) (Rel. 8)".

[2] 3GPP TR 25.996 v6.1.0, "Spatial Channel model for Multiple Input Multiple Output (MIMO) simulations," September 2003.

[3] T. Jiang,L. Song, and Y. Zhang, "Orthogonal Frequency Division Multiple Access Fundamentals and Applications," Boston MA, USA: Auerbach Publications, 2010.

[4] J. A. C. Bingham, "ADSL, VDSL and Multicarrier Modulation," 1st ed., John Wiley \& Sons, Inc., New York, NY, USA, 2000.

[5] WiMAX Forum-Technology, http://www.wimaxforum.org.

[6] Dr. Vaithiyanathan, C. Ravichandiran, "An Incisive SWOT Analysis of Wi-Fi, Wireless Mesh, WiMAX and Mobile WiMAX Technologies," IEEE (ICETC 2009), Singapore, 2009.

[7] IEEE, "IEEE standard for local and metropolitan area networks," Part 16, IEEE 802.16-2004, New York, NY, 2004.

[8] ANSI/IEEE Std 802.11, "Wireless Lan medium access control (MAC) and physical layer (PHY) specifications," 1999.

[9] H. Sari, S. Sezginer, E. Vivier, "Full Frequency Reuse in Mobile WiMAX and LTE Networks with Sectored Cells," Mobile WiMAX Symposium, 2009, MWS '09, IEEE, Oct. 2, 2009, pp. 42-45.

[10] G. Owen,"Game Theory," 3rd ed. New York: Academic, 2001.

[11] A. Goldsmith, "Wireless Communications", Stanford University, 2005.

[12] H. and Wang J., "Chunk-Based Resource Allocation in OFDMA Systems-Part II: Joint Chunk, Power and Bit Allocation," IEEE Transactions on Communications 2012, Vol. 60, No. 2, pp.499-509.

[13] Li J., Botella C. and Svensson T., "Resource allocation for clustered network MIMO-OFDMA systems," EURASIP Journal on Wireless Communications and Networking 2012, Vol. 175.

[14] Chang R. Y., Tao Z. F., Zhang J. Y. and Kuo C-C. J., "Dynamic fractional frequency reuse (D/FFR) for multicell OFDMA networks using a graph framework," Wireless Communications and Mobile Computing 2013, Vol. 13, No. 1, pp. 12-27. 
[15] Choi B., Lim S. and Lee T. J., "Sequential frequency reuse with power control for OFDMA systems," Wireless Communications and Mobile Computing 2013; Vol. 13, No. 1, pp. 37-46.

[16] M. Sinaie, and P. Azmi, "QoS-Driven Resource Allocation in Green OFDMA Wireless Networks", International Journal of Communication Systems, John Wiley \& Sons, Inc., Feb. 27, 2015.

[17] R. Giuliano , C. Monti and P. Loreti, "WiMAX Fractional Frequency Reuse for Rural Environments", IEEE Wireless Communications, Vol. 15, No. 3, pp.60 - 65, 2008.

[18] M. Rahman and H. Yanikomeroglu, "Enhancing cell-edge performance a downlink dynamic interference avoidance scheme with inter-cell coordination", IEEE Transactions on Wireless Communications, Vol. 9, No. 4, pp. 1414-1425, 2010.

[19] Stiakogiannakis I. N., Zarbouti D. A., Tsoulos G. V. and Kaklamani D. I., "Subcarrier Allocation Algorithms for multicellular OFDMA networks without Channel State Information," Proceedings of the 3rd International Symposium on Wireless and Pervasive Computing (ISWPC), Santorini, Greece, 7/9 May 2008, pp. 73-77.

[20] J. G. Andrews, A. Ghosh and R. Muhamed,"Fundamentals of WiMAX: Understanding Broadband Wireless Networking", Prentice Hall, 2007. 